\title{
Rhabdomyosarcoma of Nasopharynx
}

Sudip Kumar Das, (ENT) PGT. Medical College, Calcutta,

A. Bhowmick, Resident Surgeon,

Chittaranjan National Cancer Institute, Calcutta,

S. Mukherjee, Lecturer, Dept. of ENT Medical College, Calcutta,

L.M. Ghosh, Professor and Head,

Dept. of ENT Medical College, Calcutta,

S. Banerjee, Consultant, Dept of ENT Calcutta

Heart Clinic \& Hospital, Calcutta

\section{Abstract}

Rhabdomyosarcoma is a highly invasive malignant tumour arising from the mesenchymal tissue. Here, we reported a rare case of embryonal rhadomyosarcoma of nasopharynx with extension to different spaces of base of skull and orbit. Rarity of the case difficulty in diagnosis encourages its reporting.

\section{Key words}

Rhabdomyosarcoma. Embryonal

Rhabdomyosarcoma Nasopharynx 
T he first mention of rhabdomyosarcoma was made by Weber in Virchow's Archives (1855). This malignant tumour arising from skeletal muscle was established as separate clinical entity by the work of Stour (1946).

Horn and Enterline (1958) first classified the rhabdomyosarcoma into four subtypes - a) Alveolar b) Pleomorphic, c) Embryonic, d) Botryoids. They also observed that embryonal variety was a tumour of children and infant.

\section{Case Report}

P. M. a 6 years H. M. child was presented in ENT OPD of Medical college, calcutta ( Fig 1) with complaints of gradually increasing nasal obstruction for last 2 months, but for last 1 month obstruction became bilateral. There was history of several episodes of epistaxis within last 1 month-but it was never severe enough to seek medical help. His mother was also complaining of bulging of right eye since last 15 days along with loss of vision in the same eye. Examination revealed broadening of anterior part of right nose and

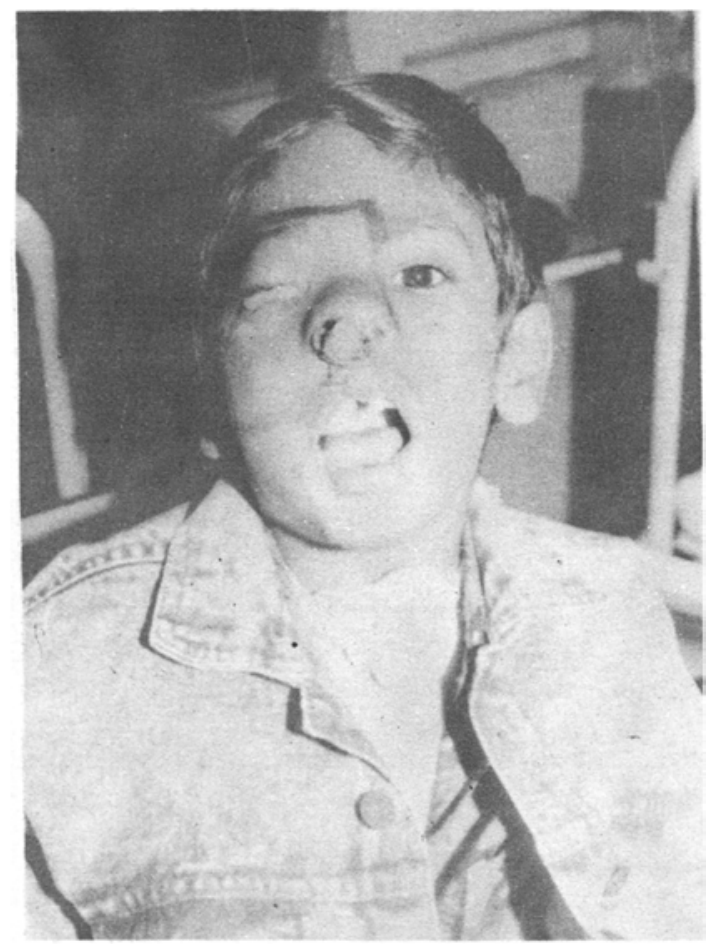

Fig 1. Clinical Photograph (Pre-Treatment) a fleshy, firm pinkish mass protruding from right nasal cavity.

It was neither friable nor bleed significantly to touch. cough impulse was negative.

Examination of oral cavity and oropharynx showed downward and forward bulging of soft palate, but hard palate and upper jaw was normal.

Anterior rhinoscopy revealed the mass occupying whole right nasal cavity. Posterior rhinoscopic examination showed a firm, fleshy lobulated, pinkish mass hanging from nasopharynx into the oropharynx obscuring the view of nasopharynx.

There was proptosis of right eye with vision reduced to P. L. lateral movement of the same eye was also absent.

No significant lymphanode was palpable.

\section{Investigations}

Routine hematological examinations were within normal limit.

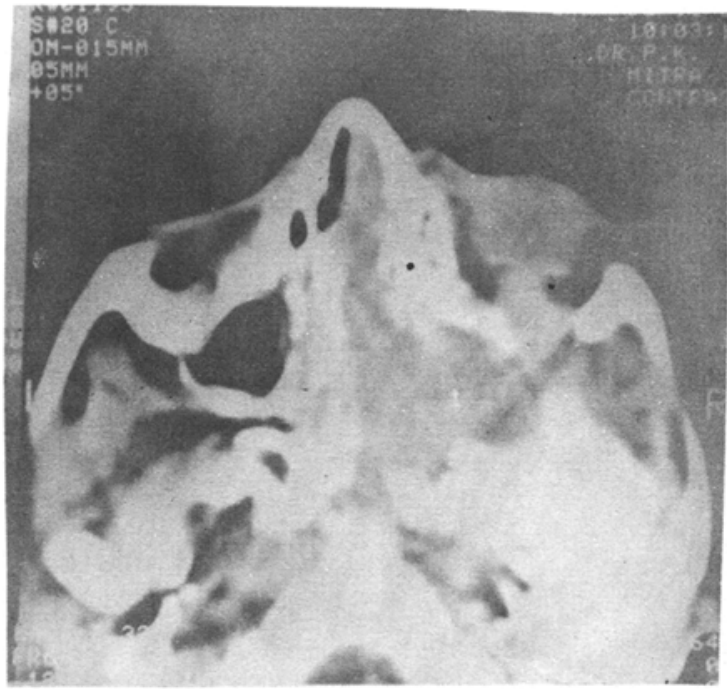

Fig 2. C. T. Sacn Showing the Tumour Mass

CT scan done to see the extension of the lesion, showed ( Fig 2) a large homogenous opacity with minimal enhancement in region of nasopharynx with extension into the region of right pterygopalatine fossa, anteriorly into right nasal cavity, laterally into 


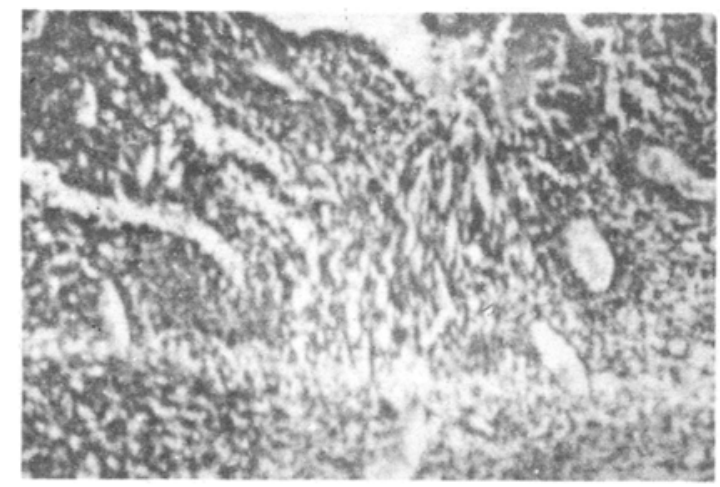

Fig 3. Histopathology (Low Power, X100) of the Tumour

infratemporal fossa, superiorly into right orbital apex, sphenoid and ethmoidal sinuses.

Biopsy was taken from the nasal mass. Histopathological examination showed (Fig 3) and (Fig 4) (low and high power) necrosis and chronic inflammation, infiltration by plasma cells, lymphocytes, and a very vascular matrix. Myxoid tissue was present in some foci. Hyperchromatic small undifferentiated round cells. some haphazardly arranged primitive mesenchymal cells were present in some foci. PAS ( Periodic acid schieff) stain also showed irregular positivity for glycogen in some cells.

Thus the diagnosis of embryonal rhabdomyosarma was made.

\section{Treatment}

During the period of doing investigations, patient developed acute respiratory distress and emergency tracheostomy had been done.

Debulking of the mass was done through lateral rhinotomy. Then patient was treated with $4000 \mathrm{cGY}$ radiotherapy and adjuvant chemotherapy. The mass regressed completely (Fig. 5) and there was no recurrence till the time of reporting.

\section{Discussion}

Rhabdomyosarcoma, an uncommon malignant tumour shows skeletal muscle differentiation and associated generally with a poor prognosis. In adult the majority of rhabdomyosarcoma are found in the extermities and in the torso. In contrast, the majority of these tumours

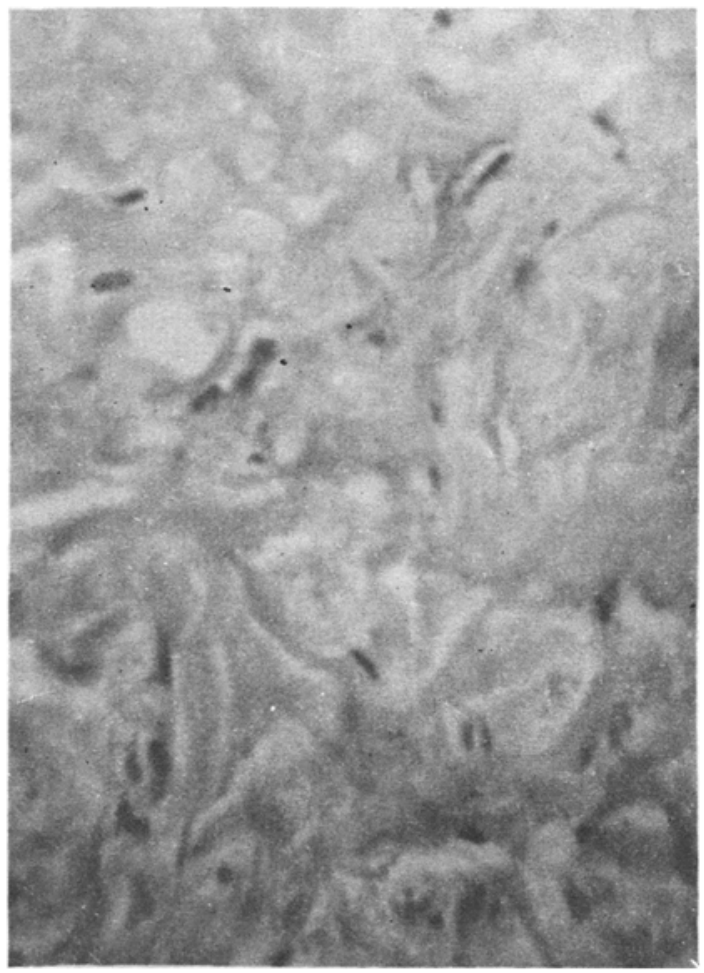

Fig 4. Histopathology (High power X400) of th I Imont

in children involve the head and neck and the urogenital tract (Stout AP and Lattes, 1970). Our case also presented with involvement of head and neck region.

FU and Perzin (1976) studied 256 non-epithelial lesion of nose, PNS and nasopharynx of which only 16 were rhabdomyosarcoma. Among these 16 cases, in only 3 cases nasopharynx was involved-one each in posterior nasal cavity and nasopharynx and oropharynx, nasopharyn $x$ and posterior oral cavity respectively.

Our patient presented with involvement of nasopharynx and nasal cavity, Dito and Bastsakis (1962) reviewed 170 reported cases of rhabdomyosarcoma of head and neck region. Of these only 16 involved the nasopharynx. The orbit was involved most frequently. In our presented case, orbit was involved.

FU and Perzin (1976) noted male; female ratio to be $5: 3$ and age of presentation ranges from 10 months to 28 years (average 7 years). Dito and Batsakis (1962) in their sudy noted that $85 \%$ of patients were below 


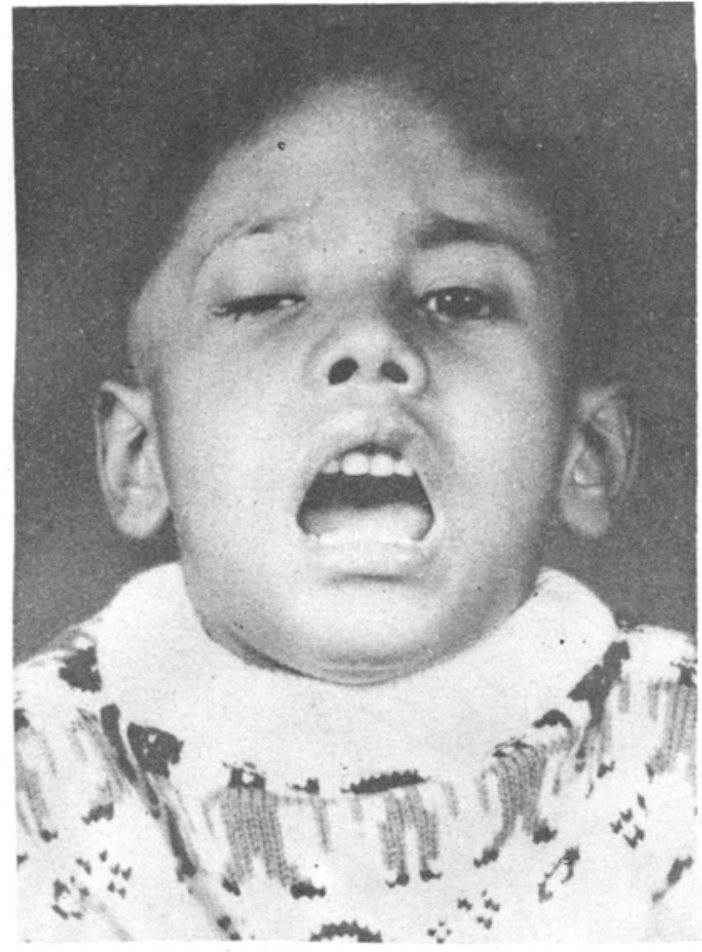

Fig 5. Clinical Photograph (Post Treatment).

20 years of age. Our presented case was a male baby of 6 years old.

In most of the cases, presenting feature was presence of a mass; followed by nasal bleeding and nasal obstruction (FU and Perzin 1976), as it was in our case.

On microscopical examination - The embry onal type of rhabdomyosarcoma showed marked cellularity containing many undifferentiated mesenchymal cells having either a spindle or round configura tions. The nucleidemonstrated hyperchromasia and variable number of mitosis. A myxomatous stroma was occasionally found in focal area (FU and Perzin, 1976)

Analysing several studies FU and Perzin (1976) concluded that peripheral rhabdomyosarcoma appear to have a some what better prognosis than those of head and neck except for the orbit which has best prognosis among head and neck lesion. Lesion of nose, PNS and Nasopharynx have a poor prognosis because of extensive local disease found at the time of diagnosis making surgical resection impossible. These tumours have already been metastasized. Most of these metastasizes is via venous channel and usually do not involve lymphnode. In our case there was no evidence of any metastasis.

Prognosis depends on histological variety. Sarcoma botryoides have a better prognosis than embryonal rhabdomyosarcoma (Sutow et al, 1970).

FU and Perzin (1976) suggested that radical surgical excision appears to be indicated whenever possible, especially those lesion growing in the nasal vestibule, ala nasi, and turbinate - as these tumors are relatively small at presentation. Preoperative radiotherapy an $\mathrm{d} /$ or chemotherapy should be used.

Where surgical resection was not possible debulking followed by radiotherapy alone or along with chemotherapy should be the ideal treatmnt as have been done in this presenting case.

\section{Conclusion}

Although rhabdomyosarcoma of head and neck region are not very common, any child with a fleshy mass which does not bleed significantly to touch, the possibility of rhabdomyosarcoma should be thought of.

\section{References}

1) Banerjee SB. Bhowmik A, Nandy TK, Ghosh LM (1994) : Embryonal Rhabdomyosarcoma of Typanomastoid region. Indian Journal of Otolaryngology and Head and Neck Surgery, 46:202-204

2) Dito WR and Batsakis JG (1962) : Rhabdomyosarcoma of the Head and Neck region Arch Surg. 84 : 582-588

3) Horn RC and Enterline HT (1958): - Rhabdomyosarcoma : a clinicopathologic study and classification of 39 cases, Cancer, $11: 187$

4) FU and Perzin (1976): Nonepithelial tumors of the nasal cavity, PNS and Nasopharynx-aclincopathologic study V. Skeletal muscle tumour. (Rhabdomyosarcoma, Cancer - $25: 1384$ - 90.

5) Sutow WW, Sullivan MP, Reed HL et al (1970) : Prognosis in childhood rhabdomyosarcoma, Cancer - 25: $1384-90$. 